
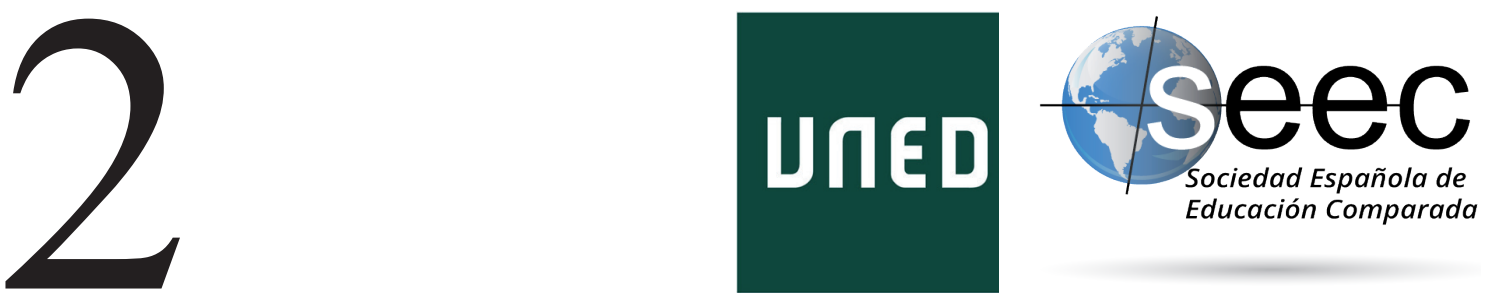

\title{
Estudio comparado del desarrollo histórico de la Psicopedagogía en Cuba y Chile
}

\author{
Comparative study of the historical development \\ of Psychopedagogy in Cuba and Chile
}

\section{Rodrigo Alberto Espinoza Vásquez*; Emilio Alberto Ortiz Torres **}

DoI: $10.5944 /$ reec.32.2018.22410

\section{Recibido: 6 de agosto de 2018 Aceptado: 28 de diciembre de 2018}

\begin{abstract}
*Rodrigo Alberto Espinoza Vásquez: Universidad Santo Tomás. Universidad Central de Chile. Centro de Estudios Psicopedagógicos Tremün, Chile. Licenciado en Educación y Profesor de Educación Diferencial, Universidad de Playa Ancha, Chile. Psicopedagogo, Instituto Profesional Los Leones, Chile. Magister en Gestión y Liderazgo Educativo, Universidad Central de Chile, Chile. Investigador en el área de la Psicopedagogía chilena, relevando el carácter histórico de ésta y proponiendo nuevas miradas en el ámbito evaluativo, cuyos fundamentos se basan en la mediación que se realiza entre evaluador y evaluado. Ha publicado artículos que van en la línea de lo descrito: Antecedentes históricos en el desarrollo de la Psicopedagogía en Chile (Pilquén, 2018), La evaluación psicopedagógica mediada como estrategia de diagnóstico vinculante (Pilquén, 2017), Entre la escolarización y el sentido de educar en Psicopedagogía: una reflexión (GESTEDU, 2016), Apuntes metodológicos en el desarrollo de evaluaciones diferenciadas (IEYA, 2016). Universidad Central de Chile. Datos de contacto: E-mail: rodrigo.espinozav@ucentral.cl, respinoza.psicopedagogia@gmail.com

**Emilio Alberto Ortiz Torres: Licenciado en Psicología. Universidad Central de Las Villas, Cuba. Doctor en Ciencias Psicológicas. Universidad Central de Las Villas, Cuba. Universidad de Holguín, Cuba. Profesor investigador de Psicología de la Universidad de Holguín, Cuba. Sus investigaciones se centran en las áreas de Psicología, Psicopedagogía, Psicodidáctica e Investigación Educativa. Cuenta con una vasta experiencia académica y destacan entre sus publicaciones: Las investigaciones sobre los estilos de aprendizaje y sus modelos explicativos (Journal of Learning Styles, 2009), La profesionalización del docente universitario a través de la investigación didáctica desde un enfoque interdisciplinar con la Psicología (Rev. Iberoamericana de Educación, 2011), Un modelo de personalidad para la formación de valores en la educación superior (2015), Una comprensión epistemológica de la Psicopedagogía (C. Moebio, 2014), entre muchas otras. Datos de contacto: E-mail: eortiz@uho.edu.cu
\end{abstract}




\title{
Resumen
}

La Psicopedagogía constituye una ciencia interdisciplinar con gran auge en los países latinoamericanos, pero son escasos los estudios que abordan su evolución histórica, de acuerdo con sus peculiaridades. El objetivo de este artículo fue realizar un estudio comparado del desarrollo histórico de la Psicopedagogía entre Chile y Cuba, dos naciones latinoamericanas que comparten un pasado y un presente peculiares, para determinar coincidencias y diferencias. Se seleccionaron como indicadores los antecedentes históricos, la institucionalización y los aportes nacionales, los cuales permitieron evidenciardichas similitudes y diferencias, de acuerdo con el desarrollo histórico, económico, cultural y político peculiar en cada país, con una evolución previa de la Psicología y la Pedagogía, como ciencias basales, a partir de las influencias de concepciones foráneas de gran arraigo en el campo educativo, pero con una labor creativa, contextualizada y exitosa de los profesionales de la Psicopedagogía, al adecuarlas a las necesidades históricas de cada país y lograr la solución científica de los problemas educativos y a la vez enriquecer dicha ciencia. La Psicopedagogía posee cierta madurez como ciencia interdisciplinar en pleno desarrollo en las condiciones actuales de Cuba y de Chile.

Palabras clave: psicopedagogía, pedagogía, psicología, Cuba, Chile

\begin{abstract}
Psychopedagogy is an interdisciplinary science with great growth in Latin American countries, but there are few studies that address its historical evolution, according to its peculiarities. The objective of this article was to carry out a comparative study of the historical development of the Psychopedagogy between Chile and Cuba, two Latin American nations that share a peculiar past and presentto determinate coincidences and differences. The historical background, institutionalization and national contributions wereselected as indicators, which allowed showing the similarities and differences according to the historical, economic, cultural and political development peculiar to each country, with a previous evolution of Psychology and Pedagogy., as basal sciences, from the influences of foreign conceptions deeply rooted in the educational field, but with a creative, contextualized and successful work of professionals in Psychopedagogy, adapting them to the historical needs of each country and achieving the solution of educational problems and at the same time enrich this science. Psychopedagogy has a certain maturity as an interdisciplinary science in full development in the current conditions of Cuba and Chile.
\end{abstract}

Key Words: Psychopedagogy, pedagogy, psychology, Cuba, Chile 


\section{Introducción}

El desarrollo que ha logrado la Psicopedagogía como ciencia interdisciplinaria, en el mundo en general y en el contexto latinoamericano en particular, ha condicionado la realización de estudios que profundizan en su evolución histórica en diferentes países, de acuerdo con sus peculiaridades nacionales y culturales. Sin embargo, son escasas las investigaciones que establecen comparaciones entre países, sobre la base de indicadores que permitan evidenciar similitudes y divergencias en esta área geográfica tan rica y diversa en lo histórico, cultural y social. Precisamente, el objetivo de este artículo fue realizar un estudio comparado del desarrollo histórico de la Psicopedagogía entre Chile y Cuba, dos naciones latinoamericanas que comparten un pasado y un presente peculiares, para determinar coincidencias y diferencias.

Para la realización de este estudio comparado se adoptó una metodología cualitativa que asume los supuestos epistemológicos planteados por González (2007),el cual defiende el carácter constructivo-interpretativo del conocimiento, en este caso con la utilización fundamental del método de análisis histórico lógico. Lo histórico y lo lógico reflejan el proceso de desarrollo en cuanto a la trayectoria temporal del objeto y su desenvolvimiento lógico, es decir, lo histórico hace referencia al movimiento del objeto en cuanto a su estructura y función, en su surgimiento y evolución posterior. Y lo lógico, en calidad de elemento derivado o como reflejo de lo histórico, determina los nexos, relaciones, determinaciones y regularidades que caracterizan ese objeto en su estado desarrollado (Castro, 2000).

La aplicación de este método histórico lógico requirió de la delimitación previa de los siguientes indicadores, que permitieron analizar los hechos cronológicos y caracterizar sus regularidades derivadas: antecedentes históricos, institucionalización y aportes nacionales. Estos indicadores facilitaron el análisis y el descubrimiento de hechos importantes, sin la pretensión de obtener resultados absolutos debido al carácter primario de este estudio.Los indicadores, como elementos concretos que permiten la evaluación empírica del objeto de estudio, se derivaron de los supuestos teóricos asumidos de una investigación histórica, que busca revelar la trayectoria progresiva peculiar que contienen los antecedentes antes de su aparición y enriquecimiento evolutivo (Ortiz, 2015).

En el caso de la Psicopedagogía, por su esencia interdisciplinar, los antecedentes históricos resultan un primer indicador indisolublemente ligado al desarrollo de la psicología y la pedagogía como ciencias basales. Posteriormente, cuando aparece realmente como institución científica profesional y como carrera universitaria, la institucionalización deviene en el segundo indicador. Su práctica científica y profesional continuada por parte de sus protagonistas, promueve la aparición de aportes nacionales que constituye el tercer indicador. Con estos tres indicadores se puede realizar un análisis comparativo inicial del desarrollo de la Psicopedagogía en Chile y en Cuba.

\section{Cuba}

\subsection{Antecedentes históricos}

Los antecedentes se pueden delimitar en el pensamiento pedagógico cubano del siglo XIX, ya que incluían de manera original, referencias a ideas psicológicas de avanzada en el mundo, influidas por las concepciones europeas que estaban en boga en esa época. 
Entre estos pensadores están Félix Varela y Morales (1788-1853) y José de la Luz y Caballero (1800-1860).

En las concepciones de estos pensadores había referencias explícitas al valor de las sensaciones en el conocimiento humano y su fuente en la realidad externa, rechazando el innatismo en el proceso formativo (Varela, 1944). El carácter educable de las aptitudes y la relación entre la cognición y lo afectivo en el desarrollo de la personalidad del educando (Luz y Caballero, 1948, 1959, 1962). Estas ideas fueron muy originales en su época, a pesar de que no poseían la necesaria sistematización y coherencia debido a su carácter especulativo, pero muy vinculadas con su ardua labor educativa, sobre todo cuando la psicología como ciencia independiente se encontraba en plena gestación.

En el período finisecular del XIX e inicios del XX se destacan los aportes de Enrique José Varona (1849-1933), por haber incorporado la enseñanza de la Psicología, ya como ciencia independiente, como asignatura: Nociones de Psicología en el plan de estudios en los Institutos de Segunda Enseñanza (bachillerato) en la naciente República de Cuba a inicios del siglo XX, así como su correspondiente libro de texto denominado Curso de Psicología, publicado en 1905 y utilizado hasta 1939, elaborado con fines didácticos que ofrece una panorámica de los conocimientos más avanzados sobre la Psicología en el mundo con fuerte influencia del positivismo (Varona, 1921).

En 1939 continúa la enseñanza la Psicología en el país como asignatura en el bachillerato, esta vez con el libro del profesor José M. Velázquez Portuondo (1901-?): Curso Elemental de Psicología, texto con gran valor metodológico por su estructura lógica y asequibilidad de los contenidos incluidos (Velázquez, 1954). Dentro de la enseñanza de esta ciencia se destacan varios psicólogos que como docentes la aplicaron al proceso formativo en los institutos de segunda enseñanza y en las escuelas normales, dedicadas a la formación de maestros primarios (Ortiz, Aguilera, Franco y Torres, 1992).

Gustavo Torroella González (1918-2006) fue el primero en aplicar la psicología en función de la educación y la orientación vocacional en varios centros escolares, incluyendo a los padres (Segura, González, González y Álvarez, 2005), a partir de los resultados obtenidos por esta ciencia en los Estados Unidos. También impartió la asignatura Psicología en el Instituto de Segunda Enseñanza del Vedado y en la Facultad de Filosofía y Letras de la Universidad de La Habana, también fue cofundador de la Escuela de Psicología (Román, 2006).

Roberto Agramonte y Pichardo (1904-1995), como jefe de la cátedra de Psicología en la Facultad de Filosofía y Letras de la Universidad de La Habana, impartía esta ciencia como asignatura a partir de su texto Tratado de Psicología General, el cual estaba influido por el conductismo (Agramonte, 1938).

Alfredo Miguel Aguayo Sánchez (1866-1949), en la Cátedra de Psicología Pedagógica, de la Escuela de Pedagogía en la Universidad de La Habana, elaboró un Tratado de Psicología Pedagógica (Aguayo, 1933) en la que define a la psicopedagogía como la psicología del niño, estudiada desde el punto de vista de la educación. Aurora García Herrera (1893-1983), en la misma cátedra, desarrolló una productiva labor docente (Dueñas, 2006) y aportó la obra Psicología Pedagógica (García, 1951), utilizada como texto en la enseñanza de dicha asignatura con contenidos actualizados, abarcadores y profundos sobre la psicopedagogía en cuanto a su concepto, división y desarrollo, los métodos y técnicas psicológicas aplicables, especialmente en los Estados Unidos y en Europa.

En esta misma cátedra de Psicología, Alfonso Bernal del Riesgo (1902-1975) elaboró con fines docentes el libro Curso Panorámico de Psicología Humana (Bernal, 1952). 
Diego González Martín (1913-1998), en su obra Didáctica o Dirección del Aprendizaje (González, 1946), realizó una fundamentación psicológica del proceso de enseñanzaaprendizaje aplicable a las condiciones de la educación en esa época. Fue cofundador también de la Escuela de Psicología de la Universidad de La Habana (González, 2004).

En este breve recorrido histórico se puede constatar que los antecedentes de la psicopedagogía estuvieron en los aportes del pensamiento pedagógico cubano del siglo XIX y en la segunda mitad del siglo XX, la labor docente e investigativa de varios psicólogos de formación, que ejercieron la docencia en diferentes niveles de enseñanza, elaboraron manuales y libros con claras intenciones didácticas, en los cuales se aplicaban explícitamente a la educación los aportes más actualizados de la ciencia psicológicas en el mundo.

\subsection{Institucionalización científica y profesional}

A partir de 1962 y 1963 se crea la carrera de Psicología en la Universidad Central de Las Villas y la Universidad de La Habana respectivamente, con la inclusión de la especialidad en Psicología Educativa, la cual tuvo una presencia importante y reconocimiento en el país en el campo investigativo y en la organización y desarrollo de diferentes direcciones en el trabajo nacional de educación (González, 2013).

A finales de la década de los años 60 se crean los Institutos Superiores Pedagógicos, cuyo origen está en las antiguas Escuelas de Pedagogía de las universidades, cuya responsabilidad era la formación profesional superior de docentes para todos los niveles de enseñanza. En los planes de estudio de todas las carreras pedagógicas se impartían asignaturas de Psicología y Pedagogía de manera independiente. También en estos institutos comienzan a desarrollar una incipiente labor investigativa en la que se aplicaba la psicología a la educación con resultados novedosos y pertinentes.

En la década del 70 se inicia la carrera de Pedagogía-Psicología dentro de los Institutos Superiores Pedagógicos, dirigida a formar especialistas de nivel superior sobre todo para que impartieran docencia, cuyo plan de estudios contenía asignaturas de ambas ciencias sin lograr la necesaria relación interdisciplinaria.

En los años 80 se crea oficialmente la figura del psicopedagogo en varios centros educacionales previos a la universidad, encargados de asesorar el trabajo educativo al nivel individual y grupal. Algunos eran graduados de la carrera de Pedagogía-Psicología pero otros no, por lo que fue necesario establecer una estrategia de profesionalización a través de cursos y diplomados que les permitieran la imprescindible idoneidad en su desempeño.

En esta misma década la Revista Cubana de Psicología, creada en la Facultad de Psicología de la Universidad de La Habana, comenzó a incluir, dentro de sus publicaciones, múltiples artículos científicos sobre psicología educativa que divulgaban resultados investigativos novedosos y pertinentes aportados por autores nacionales.

En los años 80 también se funda el Instituto Central de Ciencias Pedagógicas, adjunto al Ministerio de Educación que, como parte de su misión, desarrollan diversas investigaciones psicopedagógicas sobre las peculiaridades ontogenéticas de los alumnos, las dificultades en el aprendizaje, el desarrollo de la personalidad, del talento y la creatividad en la escuela, cuyos resultados son aplicados en todos los niveles de enseñanza.

Como resultado del análisis realizado previamente, se considera que las regularidades de la institucionalización de la Psicopedagogía en Cuba son: el desarrollo de la psicología educativa como especialización en la carrera de Psicología, la masificación y el perfeccionamiento de la enseñanza de la Psicología en la formación de profesionales de la educación, la creciente realización de investigaciones psicopedagógicas aplicadas a 
la educación y la correspondiente introducción de sus resultados a la práctica formativa en todos los niveles de enseñanza, el inicio de la influencia oficial de la psicología de orientación marxista en el país y la aparición del psicopedagogo como profesión.

\subsection{Aportes nacionales}

Varios investigadores cubanos han contribuido de manera directa al desarrollo de la Psicopedagogía como ciencia interdisciplinaria. Como resultado de la influencia de la psicología de orientación marxista en el país, a partir de los años sesenta, se comienzan a asumir sus paradigmas en la práctica profesional educativa, en las investigaciones y en la formación de pregrado y de posgrado, especialmente con aquellos profesionales de la Psicología y la Pedagogía que fueron a diferentes países socialistas europeos, sobre todo a la Unión Soviética, a realizar doctorados desde la década de los años 70 en adelante.

De manera paulatina las concepciones psicológicas aplicadas a la educación respondieron totalmente a dicho paradigma marxista, por lo que el desarrollo de la Psicopedagogía en Cuba está fuertemente influido por las diferentes interpretaciones existentes, especialmente las que tienen como sustento epistemológico el enfoque teórico general de L. S. Vigotsky (1896-1934).

Pero los psicopedagogos cubanos no se limitaron a aplicar de manera directa y descontextualizada la concepción vigotskiana, ya que la enriquecieron con sus críticas y sus aportes, a partir de miradas novedosas y complementarias a su núcleo duro, referido a la articulación de la filosofía marxista con la psicología y la pedagogía.

González y Mitjáns (1989) propusieron una comprensión más dinámica de la personalidad para su educación, con la asunción del enfoque personológico de fuerte raigambre en la Psicología Humanista proveniente de los Estados Unidos. Mitjáns (1995) abordó las relaciones internas de la creatividad, la personalidad y la educación desde este enfoque personológico, que resulta a la vez una continuidad y una ruptura con los postulados seminales de Vigotsky (Díaz y González, 2005).

Labarrere (1996) profundizó en el carácter contextual de la zona de desarrollo próximo y en la necesaria actividad metacognitiva que exige del alumno y del profesor, para que el primero sea el verdadero protagonista de su aprendizaje y de su desarrollo, que constituye un elemento novedoso a la teoría y su correspondiente adecuación al haberlo investigado en estudiantes cubanos. Álvarez (1999) aportó ideas básicas influidas por las contribuciones de la psicología y la pedagogía en la caracterización de los componentes y categorías del proceso docente, así como en la teoría del aprendizaje dentro del enfoque de la actividad.

Fariñas (2005) incorpora a la concepción vigotskiana nuevas ideas: el carácter contradictorio y dinámico del proceso de interiorización, la vivencia como unidad de análisis, la aparición y el desarrollo de las habilidades conformadoras del desarrollo personal y la inexistencia de una linealidad causal entre educación y desarrollo personal, con evidente influencia de la Psicología Humanista.

Zilberstein (2004), Silvestre (1999); Castellanos (2002), Silvestre y Zilberstein (2003) y Rico (2004), elaboraron la concepción didáctica desarrolladora y el proceso de aprender a aprender como resultado de la sistematización de varias investigaciones que venían realizando en este campo, a partir de las mejores tradiciones didácticas cubanas y de los estrechos vínculos entre el aprendizaje, la educación y el desarrollo.

Vega y Corral (2006) fundamentaron epistemológicamente el currículum universitario desde la concepción histórico cultural y Corral (1999 y 2002) la pedagogía 
universitaria, así como las potencialidades y lecturas del concepto de zona de desarrollo próximo. Y D'Angelo (2006) se ha basado en esta concepción para sus investigaciones sobre la creatividad, la educación integral y desarrolladora.

De manera que la Psicopedagogía de orientación marxista ha sido enriquecida y perfeccionada por varios investigadores cubanos, a través de su interpretación y aplicación contextualizada a las condiciones sociales y culturales nacionales, con explícitas influencias de los aportes de los psicólogos humanistas norteamericanos, lo que ha favorecido el desarrollo de esta ciencia en el país.

Sin embargo, en la formación profesional de los psicopedagogos se ha mantenido la dicotomía entre ambas ciencias, debido a que los planes de estudio siguen incluyendo asignaturas separadas que no permiten ni estimulan la integración. Incluso, el relativamente reciente inicio en el país de la especialidad de posgrado en Psicología y Pedagogía, dirigido a profesionales de la educación, no logra revertir el divorcio de dichas disciplinas y la carrera sigue denominándose Licenciatura en Pedagogía Psicología.

En la formación académica de posgrado se constata que, en el diseño de maestrías y doctorados curriculares de corte educativo, se desarrollan cursos desde concepciones psicopedagógicas en el abordaje de los fundamentos teóricos del proceso de enseñanzaaprendizaje y en los supuestos epistemológicos de la investigación educativa, lo que constituye un avance promisorio, que se refleja en tesis de maestrías y doctorales, sustentadas en estas posiciones teóricas con contribuciones teóricas y prácticas coherentes. Los egresados no solo proceden de la educación sino también de la salud, como por ejemplo, médicos y enfermeras, cuya formación posgraduada recibida le ayuda en su labor como docentes y para su práctica profesional especializada.

Por su parte, en la práctica profesional se ha arraigado el papel del psicopedagogo en equipos multidisciplinarios en la educación y en la salud pública para la atención y rehabilitación a pacientes con enfermedades crónicas y necesidades educativas especiales, lo que valida su pertinencia social y reafirma la importancia de que esta formación académica se corresponda con las exigencias sociales contemporáneas.

\section{Chile}

\subsection{Antecedentes históricos}

En el caso chileno, los intentos iniciales de construir Psicopedagogía se asocian a la inclusión de elementos propios de la psicología en el terreno pedagógico, produciéndose lo que se ha denominado la deriva psicológica en el escenario educativo (Espinoza, 2018). Si bien se registran acciones propias de la Educación Especial en Chile, cuyos hitos fundacionales propendieron a la creación de Escuelas Especiales a lo largo de todo el territorio en el siglo XIX, estas se ocupaban principalmente del déficit. El arraigo por una visión biologicista del aprender, asociado a las dificultades que en este acto ocurrían, estaban supeditados a denominar las limitaciones que niños y niñas evidenciaban a partir de su organicidad. Por ello, la creación del Laboratorio de Psicología de la Universidad de Chile en 1908, cuya pertenencia estaba asociada al Instituto Pedagógico de dicha Universidad (Salas, 1942), favoreció la aparición de investigaciones en el campo pedagógico, considerados en la obra de Guillermo Mann en el año 1911:Orientación General sobre las anomalías mentales como base de su tratamiento psicológico (Bravo y Tschorne, 1969), que buscaba dar respuesta a las dificultades evidenciadas en el proceso de enseñanza y aprendizaje en diversos centros educativos chilenos. 
Hacia 1920 se crea en la Facultad de Filosofía de la Universidad Católica de Chile un laboratorio de psicología experimental. Ambas universidades y sus respectivos laboratorios se convierten en el semillero de psicólogos y educadores.

La confluencia entre psicología y pedagogía es el resultado de una historia que llega a nuestro país, formalmente en 1925 cuando se presenta la Escala de Binet-Simon, en la cual se busca explorar elementos de cognición de orden superior: memoria, imágenes mentales, comprensión o juicio (Mora y Martín, 2007). Dicho instrumento, ideado para la evaluación de la inteligencia con fines pedagógicos (Espinoza, 2018), se diseñó para niños entre 3 y 12 años y combinaba pruebas de tipo sensoriomotriz con otras de carácter cognitivo.

Se debe mencionar que en el año 1928 también se registran estudios psicopedagógicos en la Universidad de Concepción, con la misma raigambre científica que en Santiago: un enfoque positivista. Las investigaciones se refieren a temas de expectativas vocacionales, intereses literarios en la adolescencia, predicción sobre la aptitud para el aprendizaje de idiomas extranjeros, hábitos de los niños y experiencias sexuales de los adolescentes.

Durante ese mismo año el Laboratorio de Psicología Experimental de la Universidad de Chile, funda el Centro de Investigación Pedagógica, además de diversas escuelas experimentales primarias por parte del Ministerio de Educación, cuyo fin buscaba (Salas, 1942):

- Asegurar el éxito de la experiencias pedagógicas, con base en experiencias controladas, medibles y comparables

- Formar en el profesorado criterios científicos para resolver los problemas educacionales y familiarizarlo con métodos de investigación

- Es por ello que gran parte de los logros obtenidos por las escuelas experimentales hacia 1930 se relacionan con la adaptación y normalización de instrumentos de evaluación de la inteligencia y la preparación de evaluaciones nacionales.

Estas últimas, según Salas (1942), se enfocaron en: comprensión lectora, comprensión del cálculo y razonamiento aritmético, ortografía y vocabulario, entre otras. Además, se registra la adaptación del Test de Cálculo Aritmético de Claparede, de Razonamiento Aritmético de Ballard y la prueba de Lectura Silenciosa de Anderson.

En el año 1938 el Instituto de Investigación Pedagógica de la Dirección de Educación Primaria, tomó bajo su cargo a los Gabinetes Psicopedagógicos de las escuelas experimentales. Dicho año elabora una prueba de evaluación diagnóstica para matemática, específicamente en operatoria básica con enteros, no obstante ya en 1937 había elaborado una Escala de Comprensión Lectora. Hacia 1939 realiza un ensayo metodológico de la enseñanza de la lecto-escritura para primer año de educación primaria.

El año 1941 supone un punto de partida relevante, cuando se crea el departamento de Investigaciones Psicopedagógicas en la Universidad de Chile, donde son llevados a la práctica los conocimientos obtenidos en las primeras estandarizaciones de instrumentos que evaluaban la inteligencia.

Los inicios del laboratorio de psicología centran sus esfuerzos en la realización de estudios tendientes a la evaluación y medición de la inteligencia, además de las áreas de lecto-escritura y cálculo, sirviendo como complemento pedagógico en el estudio científico del niño desde la psicología experimental. 


\section{Investigaciones sobre el aprendizaje y la enseñanza}

En el año 1929 se realizan investigaciones sobre los errores ortográficos más comunes en estudiantes que cursan educación primaria; estudio de la frecuencia de las palabras usadas de manera persistente en los tres silabarios disponibles en el país como método de enseñanza de la lecto-escritura, estudio sobre el vocabulario espontáneo usado en composiciones en niños de $3^{\circ}$ a $6^{\circ}$ de primaria y un estudio sobre el vocabulario de los adolescentes.

En 1930 se lleva a cabo una investigación cuantitativa sobre la aptitud matemática en las escuelas primarias de Santiago. Se evaluó la habilidad para el cálculo escrito aritmético y la capacidad para el razonamiento aritmético.

Investigaciones sobre el contenido de la educación.

Entre 1929 y 1937 se generan estudios enfocados en las necesidades de vida a nivel urbano y rural, a modo de proveer información para los programas de estudio que se desarrollarían. Otra investigación se orientó hacia la preparación de un vocabulario apropiado en primer grado en base a los silabarios existentes y al análisis de mil composiciones hechas por estudiantes del mismo curso, considerando la frecuencia y estructura léxica.

Investigaciones en el campo de la orientación educacional.

A partir de 1930 las Escuelas Experimentales, que funcionaban como verdaderos laboratorios pedagógicos, comienzan a investigar sobre el mejoramiento de la técnica docente. Se destaca la formación de profesores chilenos en el extranjero, principalmente en Estados Unidos y Alemania como agentes de cambio y mejora de las prácticas de enseñanza. Algunas de las investigaciones consideran: la preparación de un silabario nacional, traducciones y adaptaciones de pruebas para evaluar la inteligencia, escalas de escritura, capacidad imaginativa del escolar, encuesta al profesorado sobre educación sexual y ensayos sobre tipología escolar. En el caso de la educación secundaria se busca instalar en los profesores el uso del método científico para la solución de problemas pedagógicos. Se destacan dos estudios sobre este tema: utilización de la Escala de Sims para conocer información sobre las expectativas, retiros y vocación de los estudiantes; y la aplicación de las Pruebas Mentales A-2 y B-2, cuyo fin de orientaba en la evaluación de la inteligencia para estudiantes de primer año de humanidades, donde luego se organizaron cursos mentalmente homogéneos, con el fin de tener metodologías y programas semejantes. Sin embargo, se evidenciaron dificultades en la agrupación, ya que solo algunos presentaban una alta puntuación en rangos de inteligencia. Esto llevó a la conclusión de que los estándares usados no estaban contextualizados a la realidad chilena, pues se evaluaba bajo una métrica norteamericana, generando desconfianza en el profesorado chileno y cuestionando la validez de las evaluaciones psicométricas.

Durante este período de la primera mitad del siglo XX, la profesora Amanda Labarca (1886-1975) asume el cargo de Directora de Educación Secundaria en el Ministerio de Educación. Uno de los hechos que marcan su gestión es la apertura del Liceo Experimental Manuel de Salas en el año 1932, lugar destinado a «ensayar nuevas formas de educación y enseñanza a fin de que tales innovaciones experimentadas en un establecimiento adecuado puedan servir de base a las reformas que se intenten en la educación secundaria general» (Salas, 1942:123) En términos concretos las labores que desarrolló dicho establecimiento se enmarcan en la línea de: ensayos de métodos de enseñanza, control del trabajo escolar, planes de estudio diferenciados, programas integrados, autogobierno de los estudiantes, 
organización de servicios de orientación profesional y psicopedagogía, educación artística, investigación sobre las condiciones de educación y enseñanza, entre otros.

En el ámbito de Estudios Psicopedagógicos del Alumnado (Salas, 1942) estos se nutren en parte de los trabajos de memorias de los aspirantes al título de profesor secundario. Entre ellos se destacan: Los errores más comunes en el aprendizaje de la aritmética de Estela Estrada (1935) y Las dificultades especiales en el aprendizaje del inglés, investigación de sus causas y medidas correctivas de Isaac Vladimirsky (1936). Ambos trabajos son ejemplo de las memorias de título que los futuros profesores realizaban en el Instituto Pedagógico de la Universidad de Chile.

En octubre de 1941 se crea el Instituto de Psicología de la Universidad de Chile que entre sus departamentos contaba con el Departamento de Investigaciones Psicopedagógic as. En el caso del Laboratorio de Psicología del Instituto Pedagógico se registran extensas investigaciones a cargo de Abelardo Iturriaga (1907-1965), director en el año 1941, sobre: La imaginación de los niños de 12 años; La memoria de ideas en los niños de 12 años; La asociación libre en los niños de liceo y estudiantes de la universidad; Características de los débiles mentales ligeros; El carácter y el ambiente familiar; Rasgos de la personalidad en jóvenes y niñas de $5^{\circ}$ año de humanidades; La vida emotiva en niños normales $y$ anormales y, La coordinación motriz en niños normales y anormales.

Posteriormente, se realizan en 1944 dos estudios por parte de la Universidad de Chile cuando sale a la luz la primera publicación del Instituto de Psicología: «El vocabulario y su relación con la edad mental en el test Binet- Terman y La prueba colectiva de Inteligencia de Terman, Forma B1» (Iturriaga, 1944:314). Ambos sustentados en la validación de instrumentos que miden la inteligencia con un enfoque empirista-positivista.

Después, se registra en 1946 la fundación de la clínica psicopedagógica en la Escuela Especial de Desarrollo en la comuna de La Reina, cuya función principal era atender psicopedagógicamente a niños que presentaban grados de discapacidad intelectual y a sus familias. (Caiceo, 2010)

Ya en 1947 se crea el departamento de Psicología en la Facultad de Filosofía de la Universidad de Chile, aunque con dependencia del Instituto Pedagógico hasta el año 1959. Los futuros psicólogos tenían la posibilidad de elegir entre cuatro especialidades: Psicopedagogía, Psicología Clínica, Psicología Industrial y Criminología (Valdivieso y Tschorne, 1969:97)

En rigor, la confluencia entre educación y psicología buscaba establecer relaciones entre experiencias pedagógicas diversas y el desempeño que los estudiantes podían lograr en los test estandarizados que se aplicaban. Es necesario considerar la influencia norteamericana en este aspecto, principalmente por los estudios cursados por la profesora Irma Salas (1903-1987) en Estados Unidos, quien se convirtió en la primera mujer chilena en obtener el grado de Doctora en Educación y cuya experiencia se vio reflejada de la mano de Amanda Labarca con quien compartió labores en temáticas de innovación, gestión e investigación pedagógica.

\subsection{Institucionalización}

Tal como se indicó anteriormente, el año 1941 supone un hito en el avance investigativo de la disciplina científica, al adquirir un carácter de mayor formalidad, ya que el departamento de Investigaciones Psicopedagógicas dependía directamente de la Universidad de Chile. No obstante, se debe reconocer el aporte germinal y fundamental de las investigaciones realizadas en el Liceo Manuel de Salas y el impulso que Amanda Labarca e 
Irma Salas le asignan a la investigación pedagógica con énfasis en el conocimiento de la psicología como ciencia válida para la mejora de los procesos pedagógicos de enseñanza y aprendizaje. Tal como lo indican Bravo Valdivieso y Tschorne (1969:97), el hecho de que el desarrollo de la Psicopedagogía hubiese surgido al alero de la Universidad de Chile «con especial importancia [en] los estudios de psicología experimental» ayudaría a explicar en parte el énfasis psicodiagnóstico que ha tenido dicha profesión, principalmente a partir de la aplicación de pruebas estandarizadas para evaluar los desempeños de los estudiantes chilenos.

Entre los hechos relevantes que destacan en su desarrollo, se indica que en 1955 se crea la Clínica Psicopedagógica, dependiente del Ministerio de Educación, que se encargará de estudiar y resolver problemas de inadaptación en estudiantes de escuelas básicas. En 1957 se consigna la alianza entre la Clínica Psicopedagógica y el Servicio de Neuropsiquiatría del Hospital Roberto del Río, para estudiar y tratar a los niños disléxicos (Caiceo, 2010). Esto nuevamente demuestra el componente neurológico que estuvo presente en los primeros enfoques de la psicopedagogía y que mantiene, hasta el día de hoy, un componente biologizante que poco a poco se ha ido complementando con la inclusión de las Ciencias Sociales en el constructo de esta ciencia.

En 1965 el Ministerio de Educación, en alianza con el Hospital Luis Calvo Mackenna, crean la Escuela 15, donde destaca el trabajo de Luis Bravo Valdivieso en la atención de la dislexia. Posteriormente se integraría en un equipo multidisciplinario la profesora Mabel Condemarín (1931-2004), para ayudar en la superación de dificultades de aprendizaje de la lectura» (Caiceo, 2010:40) El aporte innegable de Condemarín, marcaría la formación de los profesionales durante más de cuatro décadas, principalmente en las áreas de lectoescritura, temáticas donde ellas profundizó sus investigaciones.

Entre 1967 y 1970, a partir del trabajo generado por la Clínica Psicopedagógica en alianza con diversos hospitales, gestionan la apertura de 44 escuelas especiales, atendiendo a niños con diversos diagnósticos, entre ellos: parálisis cerebral, dislexia, disfunción cerebral mínima, deficiencia mental, síndrome de Down, entre otros.

Hacia el año 1972 el Centro de Perfeccionamiento, Experimentación e Investigaciones Pedagógicas (CPEIP) y la Clínica Psicopedagógica organizan un curso denominado Perfeccionamiento en Dificultades de Aprendizaje, con el objetivo de que los profesores pudiesen atender de manera adecuada y pertinente a los niños y niñas que se encontraban en dicha condición (Caiceo, 2010). Ya en 1973 existían 8 Centros de Diagnóstico ligados a la Universidad de Chile, Ministerio de Educación y hospitales, además de otras de índole privada, entre ellos el Centro Psicopedagógico Holanda de Santiago.

En 1975 se produce la creación de Centros y Microcentros Psicopedagógicos, cuyo origen se encuentra en los antiguos Centros de Diagnóstico. Siete años después, dichos centros habían atendido a más de 25.000 niños en todo Chile (Caiceo, 2010)

Ya en los inicios de la década del 80 la formalización a nivel ministerial y universitario estaba en franco ascenso, considerando la implicancia de investigaciones e innovaciones asociadas a dichas políticas, entre ellas, la integración a escuelas regulares de niños con trastornos específicos de aprendizaje (Caiceo, 2010)

En diciembre de 1980, la dictadura militar de Pinochet promulga la Ley General de Universidades desde la cual se desprenden dos grandes efectos para los temas de este estudio: la pérdida del Instituto Pedagógico por parte de la Universidad de Chile, que luego se convertiría en la Universidad Metropolitana de Ciencias de la Educación, y el inicio de la privatización de la educación superior en Chile. Este hecho significó la 
desarticulación de la Universidad de Chile a través de sus sedes a lo largo del país, generando universidades regionales en las principales capitales de las distintas regiones del país. Es en una de ellas, la Universidad de Los Lagos (Osorno, X Región de Los Lagos) que siendo estatal, imparte la carrera de Psicopedagogía.

Por otra parte, el Instituto Educares, cuyo reconocimiento oficial data del 8 de octubre de 1981 y que posteriormente se transformaría en Universidad el día 15 de febrero de 1990 , se convierte en la institución pionera en otorgar el título profesional de Psicopedagogo. Este hecho ha permitido considerar que el inicio formal de la Psicopedagogía en Chile se asocie a la institucionalización y profesionalización de dicha carrera.

Desde 1983 en adelante diversas instituciones, tanto estatales como privadas, optaron por la apertura de la carrera de Psicopedagogía (Precht, 2004), abriendo una veta en el espacio educativo chileno que considera el trabajo profesional con aquellos y aquellas niños, niñas, jóvenes y adultos que se ven enfrentados a barreras en el aprendizaje y que no están asociados a discapacidad.

Los inicios de la carrera, que surgen de la formulación realizada en el Instituto Educares, cimenta sus bases en dos grandes experiencias:

1. La necesidad de reducir las brechas en aquellos niños y niñas que requieren de mayores apoyos en su aprendizaje formal y

2. El conocimiento producido en Argentina, principalmente en la Universidad del Salvador y del Colegio de Psicopedagogos de Buenos Aires, quienes en alianza con Ester Precht (1938), quien fuese Rectora del Instituto Profesional Educares y fundadora de la carrera de Psicopedagogía en Chile, donde sus contribuciones han marcado un legado que ha trascendido generaciones, permitiendo hasta el día de hoy promover una nutrida base de profesionales de la psicopedagogía en Chile, cimentan las bases de la psicopedagogía chilena actual. En ese entonces se plantea el perfil del Psicopedagogo como:

\begin{abstract}
El profesional que con una sólida formación psicológica, pedagógica y de salud, es capaz de prevenir, detectar y corregir precozmente, alteraciones del desarrollo, crecimiento y madurez que dificultan la adquisición de los aprendizajes humanos, comprendidos entre el nacimiento y la adolescencia, señalando que el futuro profesional tendrá una formación teórica y práctica que le permita con conocimiento y creatividad, adaptarse a las situaciones del momento y al mismo tiempo, proyectarse para hacer frente con eficacia a las diferentes situaciones que puedan surgir en el futuro en el ejercicio de su profesión. (Precht, 2004:6)
\end{abstract}

El Instituto Educares funcionó en tres ciudades: Santiago, Viña del Mar y Rancagua. En cada una de sus sedes puso en marcha los Centros de Atención Psicopedagógica, apoyados por alumnos en fase final de la carrera y donde se atendía a la comunidad correspondiente.

Una vez que el Instituto se transforma en Universidad, creándose la carrera de Psicología, se abre el CAPSI, Centro de Atención Psicológica y Psicopedagógica, donde se atendían a estudiantes de toda la institución (Precht, 2004)

A partir de 1993 se conforma el comité académico de la Universidad Educares, que producirá cuatro publicaciones: Una reflexión preliminar (1995), Teoría Psicopedagógica: Extensión y Profundización (1996), El hecho Psicopedagógico (1997) y Hacia una Psicopedagogía Comunitaria (1999), que sirvieron de base para el trabajo posterior en la formación de psicopedagogos en el país. 
A nivel internacional, en 1984 se crean la Jornadas Trasandinas de Aprendizaje (Precht, 2015), impulsadas por Ester Precht de Chile y Elizabeth Calvo de Argentina, quienes buscan establecer a nivel latinoamericano una red de psicopedagogos que incluye a profesionales de Perú, Argentina, Chile, Paraguay, Uruguay y Brasil, donde comparten experiencias, investigaciones y avances en el terreno educativo.

\subsection{Aportes nacionales}

El proceso evolutivo que vivió el desarrollo psicopedagógico en Chile, transitó inicialmente desde una veta norteamericana (Salas, 1942), principalmente debido a que los primeros estudios de posgrado en el extranjero en el área educativa -en particular los casos de Amanda Labarca e Irma Salas- fueron en dicho país a inicios del siglo XX. Sabido es que había una fuerte presencia de la Psicología Experimental, con carácter cuantificante y empirista, cuyas transferencias a la realidad chilena no fueron aplicables, debido a la idiosincrasia y características propias de la nación latinoamericana, que distaba mucho del momento histórico y cultural de E.E.U.U. Dicho enfoque chocaba con la educación prusiana (Letelier, 1885) que el país había adoptado y cuyo mayor exponente se encontraba en la formación de profesores en el Instituto Pedagógico.

El enfoque norteamericano intentaba ingresar a las aulas, pero fue la propia realidad chilena que terminó por demostrar que un trasplante cultural de este tipo era poco viable. Posterior a esto y avanzando hacia la década de los 50, se hace presente el enfoque de la Epistemología Genética de Piaget, que permitió diversificar la perspectiva dominante: el conductismo, pero cuyas raíces aún están arraigadas en las aulas. Ambos enfoques disponían de una serie de instrumentos de evaluación, donde también divergían enfoques disímiles. La corriente conductista optaba fuertemente por la cuantificación y cosificación de los aprendizajes, principalmente por la necesidad de demostrar los efectos que tenían los estímulos dispuestos por los educadores en el acto de enseñar y, supuestamente, en el aprender del estudiantado. La Psicología Genética optaba por la cualificación de los aprendizajes, interpretando y categorizando a los estudiantes en diversos estadios cognitivos, según sus edades y logros asociados.

Luego, ya en los años 90 cuando se produce la reforma educativa (Donoso, 2005) del gobierno de Frei Ruiz-Tagle, surge como novedad el constructivismo y de la mano, el nombre de L. Vigotsky. No obstante y quizás debido al proceso de transición hacia la democracia luego de la dictadura de Pinochet, es que la visión socio-histórica del aprendizaje de dicho autor es estudiada y entendida de manera aséptica, es decir, paradójicamente se descontextualiza y se vuelve ahistórica, ya que prácticamente no existen menciones al carácter marxista de la propuesta de Vigotsky y, se termina minimizando y reificando la obra del autor a un único tema que es la Zona de Desarrollo Próximo. El legado de Vigotsky, en Chile, se termina por asumir como una «receta» para el enseñar y el aprender.

Estos tres grandes enfoques: el conductismo, la psicología genética constructivista y el constructivismo socio-histórico conviven actualmente en Chile. El proceso de cimentación ha sido en espiral y a medida que se despercude el conocimiento de uno, surge el otro, pero con una mirada tecnocrática, transaccional y bancaria. La lectura de los tiempos y espacios, de las necesidades y asertividades propias de un país tan diverso como Chile, han impactado, sin duda, en cómo se ha hecho Psicopedagogía. Llamativo resulta que al ser y tener un componente institucional a partir de lo que se ha hecho en Argentina, los enfoques sean tan diversos, pues en Chile el componente psicoanalítico 
(González, 2016) del aprender prácticamente no existe. Como lo expresa Peña (2014) de todos modos cualquier constructivismo tiene poco espacio para desarrollarse, principalmente por el carácter neoliberal del sistema educativo chileno.

En todo este contexto y en los años que han transcurrido - prácticamente un sigloha habido avances que provienen de diversos profesionales, no necesariamente psicopedagogos, para la construcción de esta disciplina científica.

Irma Salas y Amanda Labarca (1932) desarrollan en conjunto la experimentación psicopedagógica a partir de las necesidades de actualización que requiere la educación chilena. Comienzan los primeros intentos evaluativos integrales para comprender el proceso de aprendizaje en estudiantes chilenos de manera experimental.

Abelardo Iturriaga (1944) genera estudios ad hoc a situaciones problemáticas en el aprender, cuya diversidad no solo se limita a cuestiones curriculares, sino que opta por la indagación socio-emocional en los estudiantes.

Luis Bravo Valdivieso (1969) desarrolla estudios en el componente psicolingüístico del aprendizaje, asociado a la profundización en la comprensión de la dislexia. El desarrollo de este y otros trabajos, que posteriormente realizó con Arturo Pinto, develan la necesidad de comprender las dificultades de aprendizaje desde el lenguaje y el pensamiento.

Mabel Condemarín (1978) se instala como un referente esencial en proceso de intervención (acompañamiento) psicopedagógico, pues sus estudios en conjunto con María Elena Gorostegui y Mariana Chadwick en Madurez Escolar (1978, $1^{\circ}$ ed.), se transformó en un pilar fundamental de la comprensión de las Necesidades Educativas Especiales desde una mirada cognitiva y contextual, dándole predominancia a estos componente como los grandes alicientes del aprender.

Ester Precht (2004) cimenta las bases institucionales de la profesión en la década de los 80, pues el desarrollo germinal de las redes creadas con Psicopedagogas de Argentina, dan pie para crear el título de Psicopedagogo, vigente hasta el día de hoy, tal como ocurre con la Jornada Trasandinas.

Roberto Careaga (1997) se convierte en uno de los referentes principales del desarrollo teórico-práctico del quehacer psicopedagógico hasta la actualidad. Sus textos han permitido establecer un puente entre el desarrollo profesional y la comprensión de las barreras para acceder a los aprendizajes formales.

Jaime Bermeosolo (2005) presenta una mirada epistemológica sobre la psicopedagogía, enfocada en el quehacer de este. Plantea que en Chile se ha limitado su accionar hacia las NEE y las dificultades de aprendizaje asociadas al contexto escolar, por lo que propone que se genere un salto cualitativo en este sentido y evolucione hacia un enfoque más integral, donde pueda constituirse como metateoría respecto del cómo aprendemos los seres humanos.

\section{Conclusiones}

El estudio comparado del desarrollo histórico de la Psicopedagogía entre Cuba yChile, sobre la base de los indicadores seleccionados: antecedentes históricos, institucionalización y aportes nacionales, permitió identificar las regularidades de la evolución de esta ciencia interdisciplinar en cada país, en las que se detectan coincidencias y diferencias notorias,de acuerdo con el desarrollo histórico, económico, cultural y político peculiar en cada país.

El desarrollo de la Psicología y de la Pedagogía, como disciplinas basales, constituyó un antecedente histórico común en su aplicación directa y expedita en el campo educativo, 
sobre la base de los resultados de investigaciones empíricas y del ejercicio profesional, especialmente en las dificultades y trastornos del aprendizaje, como parte de la atención a las diferencias individuales. En ambas naciones se aprecia la incipiente influencia norteamericana en los inicios del siglo XX, cuyas acciones se asocian a la compresión del fenómeno educativo desde la psicología. Las líneas temáticas se instalan bajo un paradigma positivista, que deviene en conductismo. Asimismo se comienzan a generar investigaciones en ambos contextos nacionales, cuyas temáticas acercan la psicología a la pedagogía.

En la institucionalización de la Psicopedagogía fueron evidentes las influencias de concepciones psicológicas foráneas de fuerte arraigo en el campo educativo, que en caso de Cuba fue el predominio inicial y oficial de la concepción vigotskiana y en el caso de Chile fueron las concepciones conductistas, la corrientes de la psicología humanista y la concepción piagetana, tanto en las investigaciones empíricas, como en la formación profesional de los psicopedagogos. Hay que destacar la influencia del desarrollo de la Psicopedagogía en Argentina. Ni en Cuba ni en Chile la concepción psicoanalítica tuvo una influencia importante. También es destacable que en ambas naciones el desarrollo de la Psicología tuvo un vínculo estrecho con la formación de profesores, aunque se releva que en el caso chileno fue previo al cubano. No obstante, la historia política de ambos países difiere en le permeabilidad de la corriente marxista de la psicología y la educación, cuya influencia es mucho más evidente en Cuba. En el caso chileno, la psicología vigotskiana ingresa tardíamente, pues una vez recuperada la democracia en 1990 se comienza un proceso de reforma educacional que considera los aportes de la escuela soviética, pero que son estudiados asépticamente, desenraizados de su corriente marxista. Esto devela que la aplicabilidad en Cuba y Chile de este enfoque difiera en el contexto educativo. Políticamente hablando, ambas naciones se encuentran en las antípodas de la concepción político-económica, ya que en el caso cubano la educación es parte esencial de los derechos civiles; a diferencia de lo que ocurre en Chile, cuya comprensión neoliberal de la educación la constituye como bien de consumo. A su vez, es relevante considerar que el desarrollo cubano de la Psicopedagogía se aprecia en su inicio como una profesión que combina ambas ciencias - psicología y pedagogía - e incluso, la denomina como tal. En Chile, esto logra inicialmente considerarse como una mención de especialidad en Psicología, para luego en los años 8o, se constituya como carrera profesional. Un punto de convergencia relevante es que en ambas naciones la Psicopedagogía es concebida como una disciplina científica cuyo objeto de estudio es la comprensión de la situación de aprendizaje en diversos contextos, asociado al apoyo requerido para minimizar las barreras identificadas en dicho proceso.

Los aportes nacionales demuestran la labor creativa, contextualizada y exitosa de los profesionales de la Psicopedagogía, al adecuar las concepciones foráneas a las condiciones contextuales históricas, sociales y culturales peculiares de cada país, lo que permitió la solución científica de los problemas educativos y a la vez el enriquecimiento teórico de esta ciencia.

Se puede afirmar que la Psicopedagogía en ambos países posee la madurez científica suficiente, para que los resultados de las investigaciones realizadas sean novedosos y pertinentes, la práctica profesional es rigurosa y científicamente fundamentada y en la formación de profesionales se refleja esta madurez, aunque todavía de forma insuficiente.

El carácter exploratorio de este trabajo obliga a considerar estas conclusiones como preliminares, por lo que se hace necesaria la continuidad de este estudio para el logro de la obligatoria profundización y la necesaria certeza científica de los resultados obtenidos. 


\section{Referencias}

Abarca, A. (1939) Historia de la enseñanza en Chile. Chile: Publicaciones de la Universidad de Chile, Universitaria.

Agramonte, R. (1938) Tratado de Psicología General. Dos tomos. La Habana: Edición Cultural SA.

Aguayo, A. (1933). Tratado de Psicología Pedagógica. La Habana: La Moderna Poesía.

Álvarez, C. (1999). La Escuela en la Vida. La Habana: Pueblo y Educación.

Bermeosolo, J. (2005) Cómo aprenden los seres humanos. Una Aproximación Psicopedagógica. Chile: Ediciones UC.

Bernal del Riesgo, A. (1952). Curso Panorámico de Psicología Humana. Dos tomos. La Habana: Edición Cuarta Provisional.

Bravo Valdivieso, L. y Tschorne Tetelman, P. (1969). La Psicología en Chile. Revista Latinoamericana de Psicología, 1(2-3), pp. 95-104. Recuperado el 5 de mayo de 2015 desdehttp://www.redalyc.org/articulo.oa?id=80501301

Caiceo, J. (2010) Esbozo de la educación especial en Chile: 1850 - 1980. Revista Educación y Pedagogía, 22(57). Recuperado el 23 de marzo de 2015 desde http://aprendeenlinea.udea.edu.co/revistas/index.php/revistaeyp/article/ viewFile/9839/9038

Careaga, R. (1997) El hecho psicopedagógico. Investigación e Intervención. Chile: Universidad Educares-Bravo y Allende Editores.

Castellanos, D. (2002). Aprender y enseñar en la escuela. La Habana: Editorial Pueblo y Educación.

Castorina, J. (2016). Algunos problemas epistemológicos de la teoría psicológica y de la práctica psicopedagógica. Pilquen, Sección Psicopedagogía, 13(2).

Castro, Luis. (2000). Diccionario de ciencias de la educación superior. Lima: Seguro editores.

Condemarín, M. Chadwick, M., Gorostegui, M. y Milicic, N. (1978) Madurez Escolar. Chile: Andrés Bello.

Corral, R. (1999). Las «lecturas» de la zona de desarrollo próximo. Revista Cubana de Psicología, 16(3), pp. 1-6, La Habana. Recuperado de: http://pepsic.bvsalud.org/ $\mathrm{pdf} / \mathrm{rcp} / \mathrm{v} 16 \mathrm{n} 3 / \mathrm{o} 7 . \mathrm{pdf}$

Corral, R. (2002). La zona de desarrollo próximo y la pedagogía universitaria, Revista Temas, 31, pp. 27-32, La Habana.

D’Angelo, O. (2006). La Complejidad y la Educación Integradora y Desarrolladora: El Currículo PVAI. CIPS. La Habana: Academia de Ciencias de Cuba. 
Decreto con fuerza de ley ${ }^{\circ}{ }^{1}$. Fija Normas Sobre Universidades de $1980 § 3.541$

Díaz, Á. y González, F. (2005). Subjetividad: una perspectiva histórica cultural. Conversación con el psicólogo cubano Fernando González Rey. Univ. Psychol. Bogotá, 4(3), pp. 373-383, octubre-diciembre. Recuperado de: http://sparta. javeriana.edu.co/psicologia/publicaciones/actualizarrevista/archivos/ V4N310subjetividad.pdf

Donoso, S. (2005). Reforma y política educacional en Chile 1990-2004: El neoliberalismo en crisis. Estudios Pedagógicos 31(1), pp. 113-135.

Dueñas, J. (2006). Doctora Aurora García Herrera (1893-1983): primera rorscharchista cubana. Revista del Hospital Psiquiátrico de la Habana 3(3), pp. 1-4.

Espinoza Vásquez, R. A. (2018). Antecedentes históricos en el desarrollo de la Psicopedagogía en Chile. Pilquén, Sección Psicopedagogía, Vol. 15, $N^{\circ} 1$.

Fariñas, G. (2005). Psicología, educación y sociedad. Un estudio sobre el desarrollo humano. La Habana: Editorial Félix Varela.

García Herrera, A. (1951) Psicología Pedagógica. La Habana: Editorial Cultural, S.A.

González, L. (2016) Nuevos pacientes...Una clínica psicopedagógica posible. Pilquen Sección Psicopedagogía, 13(2).

González Martín, D. (1946). Didáctica o Dirección del Aprendizaje. La Habana: Editorial Cultural.

González Serra, D. (2004). La obra científica de Diego González Martín. Revista Cubana de Psicología, 21(3), pp. 249-259. Recuperado: http://www.dict.uh.cu/rev_ ps_2004_no3.asp

González Rey, F. y Mitjáns, A. (1989). La personalidad: su educación y desarrollo. La Habana: Editorial Pueblo y Educación.

González Rey, F. (2007) Investigación cualitativa y subjetividad: los procesos de construcción de la información. México, D.F: Mc Graw-Hill Interamericana.

González Rey, F. (2013). Sociedad y psicología en Cuba: avanzando en uno de sus caminos de investigación y construcción teórica durante tres décadas. Revista Interamericana de Psicología, 47(2), pp. 195-210. Recuperado de: http://www. redalyc.org/pdf/284/28430082005.pdf

Iturriaga, A. (1944) Archivos del Instituto de Psicología, Vol. I. Chile: Prensas de la Universidad de Chile.

Labarrere, A. (1996) Pensamiento. Análisis y autorregulación de la actividad cognoscitiva de los alumnos. Pueblo y Educación: La Habana.

Letelier, V. (1885) Las Escuelas de Berlín. Informe elevado al supremo gobierno por la legación de Chile en Alemania. Santiago: Imprenta Nacional Moneda. 
Luz y Caballero, J. (1948). La polémica filosófica. La Habana: Editorial de la Universidad de La Habana.

Luz y Caballero, J. (1959). Elencos y discursos académicos. Editorial de la Universidad de La Habana.

Luz y Caballero, J. (1962). Aforismos. La Habana: Editorial de la Universidad de La Habana.

Ministerio de Educación, Chile (2016). www.portales.mineduc.cl/usuarios/sies/File/.../ IESCERRalo9112010web1.xls. Recuperado el 28 de junio de 2016

Mitjáns, A. (1995). Creatividad, Personalidad y Educación. La Habana: Pueblo y Educación.

Mora, J. y Martín. M. (2007) La escala de inteligencia de Binet y Simon (1905) su recepción por la Psicología posterior. Revista de Historia de la Psicología, 28(2-3).

Ortiz Torres, E. Aguilera, M.; Franco, I. y Torres, N. (1992). El desarrollo de la psicología en Holguín desde 1902 hasta1959. Revista Cubana de Psicología, 9(2), pp. 109113. Universidad de La Habana.

Ortiz Torres, E. (2015) La dialéctica en las investigaciones educativas. Manta, Manabí, Ecuador: Colección Tiempos de Aprender. Editorial Mar Abierto.

Peña, M. (2014) Una mirada a la teoría del conocimiento de Jean Piaget, a 20 años de la llegada del constructivismo a la educación chilena. Revista Inclusiones, 1(4).

Precht, E. (2015) Recopilación de antecedentes jornadas trasandinas 1984 - 2015. UNESCO, Santiago. Chile.

Precht, E. (2004) Inicio y evolución de la Carrera de Psicopedagogía en Chile. Seminario Calidad de la Educación en el Contexto de un Mundo Globalizado. X Jornadas Trasandinas

Rico, P. (2004). Proceso de enseñanza-aprendizaje desarrollador en la escuela primaria. La Habana: Editorial Pueblo y Educación.

Román Hernández, J. (2006). Gustavo Torroella González-Mora (1918-2006): pionero de la Psicología en Cuba. Revista Latinoamericana de Psicología, 38(3), septiembre-diciembre, pp. 621-625, Bogotá. Recuperado de: http://www.redalyc. org/pdf/805/80538312.pdf

Salas, I. (1942) La investigación pedagógica en Chile. Anales de la Universidad de Chile, 1941.

Segura, M. E.; González, D.; González, M.E. y Álvarez, M. I. (2005). Teorías psicológicas y su influencia en la educación. La Habana: Editorial Pueblo y Educación.

Silvestre, M. (1999). Aprendizaje, educación y desarrollo. La Habana: Editorial Pueblo y Educación. 
Silvestre, M. y Zilberstein, J. (2003). Hacia una didáctica desarrolladora. La Habana: Editorial Pueblo y Educación.

Varela, F. (1944). Miscelánea Filosófica. Editorial de la Universidad de La Habana.

Varona, E. J. (1921). Curso de Psicología. Segunda Edición. La Habana: librería e imprenta La Moderna Poesía.

Valdivieso, L. y Tschorne, P. (1996) La Psicología en Chile. Revista Latinoamericana de Psicología, 1(2-3).

Vega, R. y Corral, R. (2006). La fuente epistemológica del currículum, referente imprescindible en el diseño de una carrera dirigida a la investigación científica. Revista e-Currículum, diciembre, Año 2, 2(1), Pontificia Universidad Católica de Sao Paulo. Recuperado de: www.puesp.br/ecurriculum

Velázquez, J. M. (1954). Curso Elemental de Psicología. Sexta edición revisada. La Habana: editorial Minerva.

Zilberstein, J. (2004). Aprendizaje desarrollador. La Habana: Editorial Universitaria. 